\title{
Fully solution processed liquid metal features as highly conductive and ultrastretchable conductors
}

\author{
Hangyu Zhu ${ }^{1}$, Shaolei Wang ${ }^{1}$, Menghu Zhang ${ }^{1}$, Tingyu $\mathrm{Li}^{1}$, Gaohua Hu${ }^{1}$ and Desheng Kong $\mathbb{D}^{1 凶}$
}

Liquid metal represents a highly conductive and inherently deformable conductor for the development of stretchable electronics. The widespread implementations of liquid metal towards functional sensors and circuits are currently hindered by the lack of a facile and scalable patterning approach. In this study, we report a fully solution-based process to generate patterned features of the liquid metal conductor. The entire process is carried out under ambient conditions and is generally compatible with various elastomeric substrates. The as-prepared liquid metal feature exhibits high resolution $(100 \mu \mathrm{m})$, excellent electrical conductivity $\left(4.15 \times 10^{4} \mathrm{~S} \mathrm{~cm}^{-1}\right)$, ultrahigh stretchability (1000\% tensile strain), and mechanical durability. The practical suitability is demonstrated by the heterogeneous integration of light-emitting diode (LED) chips with liquid metal interconnects for a stretchable and wearable LED array. The solution-based technique reported here is the enabler for the facile patterning of liquid metal features at low cost, which may find a broad range of applications in emerging fields of epidermal sensors, wearable heaters, advanced prosthetics, and soft robotics.

npj Flexible Electronics (2021)5:25; https://doi.org/10.1038/s41528-021-00123-x

\section{INTRODUCTION}

The rapid expansion and evolution of wearable technology have stimulated the growth of stretchable electronics ${ }^{1-4}$. The compliant mechanical properties allow the intimate and stable integration of functional devices with the human body for a broad range of emerging fields, including health monitoring systems ${ }^{5-8}$, robotic prosthetics $^{9,10}$, and human-machine interfaces ${ }^{11-13}$. Among various building components, intrinsically stretchable conductors present a class of enabling materials for soft forms of devices with inherent deformability ${ }^{14}$. The improved stretchability may further allow the heterogeneous integration of conventional rigid components over elastomeric substrates, thereby achieving mechanical deformability and electronic functionality on the circuit level ${ }^{15,16}$. Intrinsically stretchable conductors have been extensively explored in terms of soft nanocomposites ${ }^{17-19}$, conducting polymers ${ }^{20-22}$, hydrogels ${ }^{23,24}$, and conductive liquids ${ }^{25-27}$. Liquid-state conductors confined in elastomers stand out as attractive material candidates with the ultimate deformability merely limited by the fracture strains of the encapsulants ${ }^{26,28}$. Galinstan, a Ga-based ternary liquid metal alloy, further exhibits high electrical conductivity $\left(3.46 \times 10^{4} \mathrm{~S} \mathrm{~cm}^{-1}\right)$, low melting point $\left(-19^{\circ} \mathrm{C}\right)$, and non-toxicity ${ }^{29,30}$. These desirable attributes allow Ga-based liquid metal to be adopted in a variety of stretchable electronic devices and systems, such as antenna ${ }^{31,32}$, wearable sensors ${ }^{33-36}$, epidermal heaters ${ }^{33,37}$, and integrated circuits ${ }^{15,16}$.

A key step towards functional devices in deformable form factors requires the patterning of liquid metal into well-defined features. An intuitive approach utilizes the liquid characteristics of gallium-based alloys for the injection into embedded microfluidic channels in elastomers ${ }^{38-42}$. The manufacturing challenges and cost issues involved in the fabrication of soft microfluidic devices with delicate microchannels represent the major limitation of this patterning process ${ }^{43}$. Complicated design considerations are also required for the embedded liquid metal to interface with other materials components during device fabrication ${ }^{26}$. The direct ink writing of liquid metal harnesses spontaneously formed native oxides to stabilize printed features and thereby enables maskless fabrication of arbitrary patterns, which unfortunately requires delicate control over the printing parameters and selected substrate with suitable properties ${ }^{42,44,45}$. Liquid metal nano/ microparticles through top-down synthesis are highly solution processible and readily formulated into various inks compatible with scalable printing techniques ${ }^{46-49}$. As printed features often exhibit poor electrical conductivity and therefore require additional sintering treatments $37,50-52$. A promising approach harnesses the reactive wetting of non-oxidized liquid metal towards $\mathrm{Au}$ and $\mathrm{Cu}$ film templates to enable scalable and robust fabrication of delicate patterns $15,32,53,54$. The process, on the other hand, demands advanced microfabrication facilities for physical depositions of solid metal films and subsequent patterning into highfidelity features over elastomeric substrates. Special care is often taken to prevent the catastrophic breakdown of the film due to the compressive strains built during the deposition processes with elevated temperatures ${ }^{55,56}$, which requires additional efforts to develop tailored recipes for individual elastomers ${ }^{32}$.

In this study, we report a scalable and low-cost fabrication approach to create fully solution-processed liquid metal features directly over elastomeric substrates, which exhibits fine pattern resolution $(100 \mu \mathrm{m})$, excellent electrical conductivity $(4.15 \times$ $\left.10^{4} \mathrm{~S} \mathrm{~cm}^{-1}\right)$, ultrahigh stretchability $(1000 \%$ tensile strain), and mechanical durability. Screen-printed sacrificial masks define the locations and morphologies of desired features over the elastomer substrate. A layer of polydopamine (PDA) is then deposited by self-polymerization of dopamine solution as a universal surface functionalization to enable electroless plating of Cu films. As-prepared Cu pattern functions as the template to create liquid metal feature through reactive wetting. The overall process is carried out under the ambient conditions without advanced capital equipment and allows the facile preparation of

${ }^{1}$ College of Engineering and Applied Sciences, State Key Laboratory of Analytical Chemistry for Life Science, and Jiangsu Key Laboratory of Artificial Functional Materials, Nanjing University, Nanjing 210046, China. ${ }^{凶}$ email: dskong@nju.edu.cn 
a
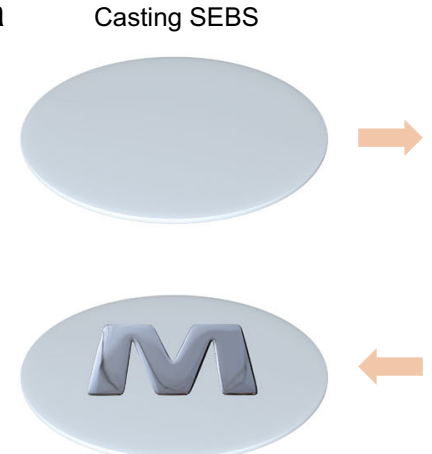

Liquid Metal Pattern
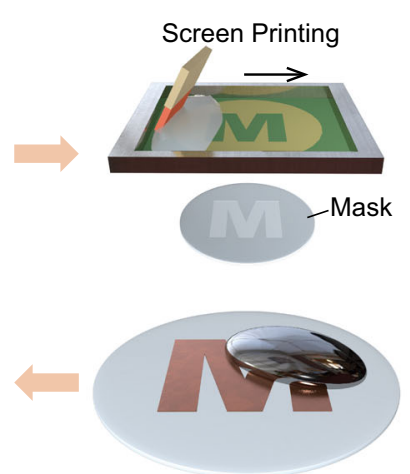

Coating Liquid Metal
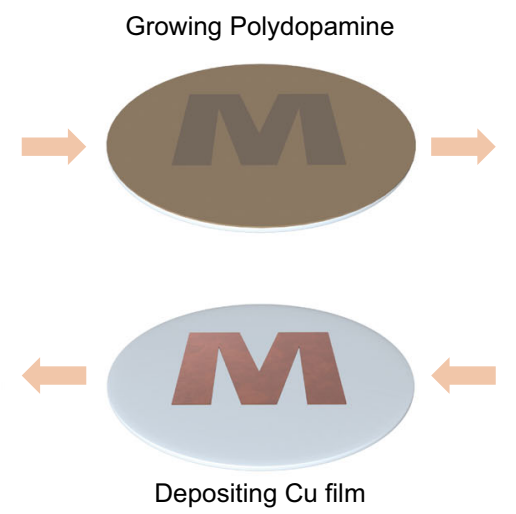

Depositing Cu film

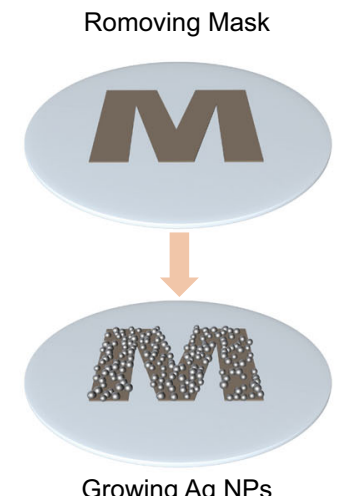

Growing Ag NPs
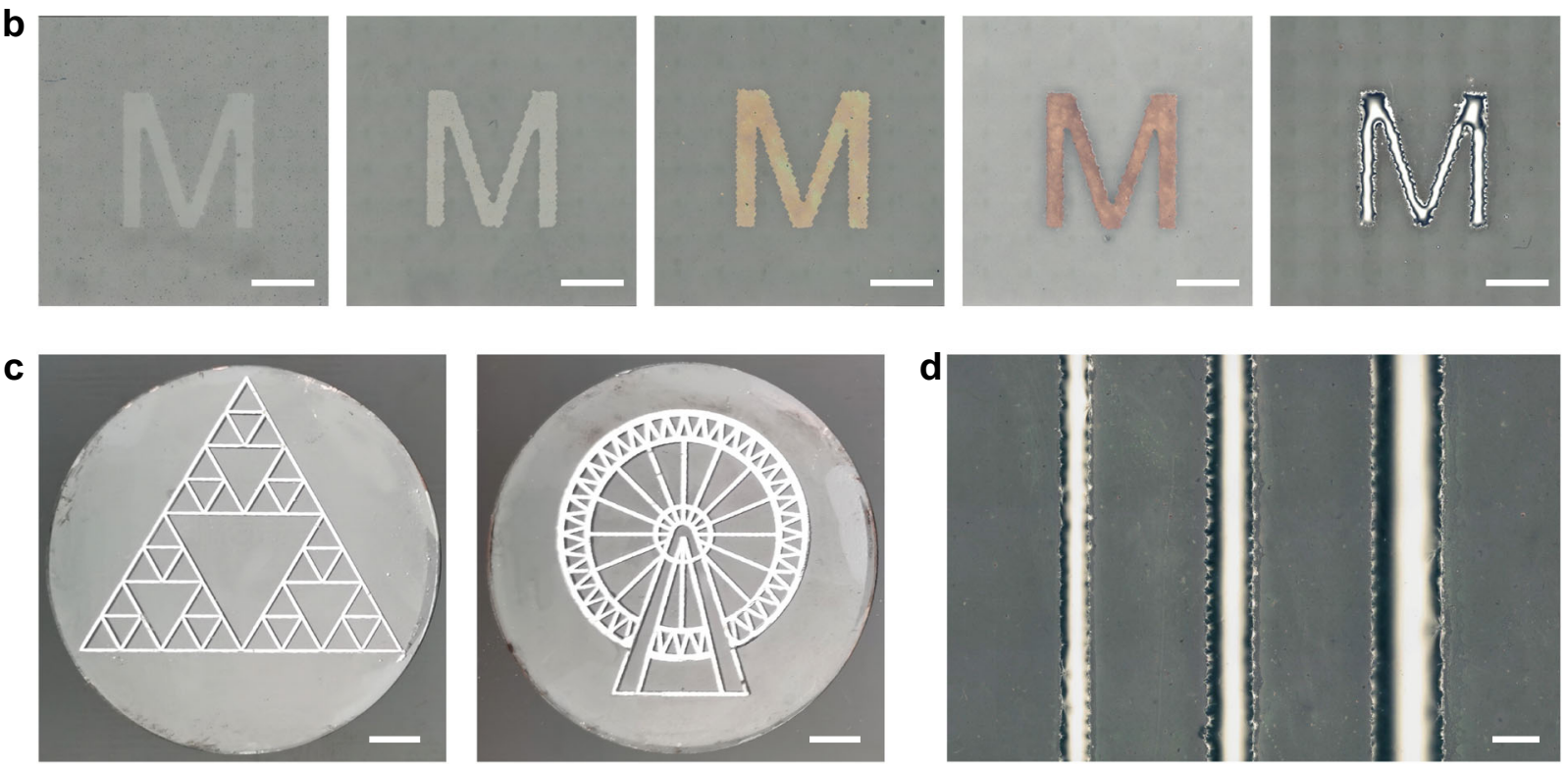

1

Fig. 1 Liquid metal patterning via solution-based processes. a Schematic illustration of the process flow to create patterned liquid metal on SEBS elastomer substrate. b Corresponding optical microscopy images at the key steps including (from left to right) screen-printed shadow mask, patterned polydopamine (PDA) layer, deposited Ag nanoparticles (NPs), electroless plated Cu film, and liquid metal pattern. Scale bar: $1 \mathrm{~mm}$. c Optical images of as-prepared liquid metal patterns in the shape of Sierpinski triangle (left) and sky wheel (right) on SEBS substrates. Scale bar: $1 \mathrm{~cm}$. d Optical microscopy image of an array of line-shaped patterns to reveal the feature resolution. Scale bar: $200 \mu \mathrm{m}$.

liquid metal patterns over a variety of elastomers, including styrene-ethylene-butylene-styrene thermoplastic polyurethane (SEBS), thermoplastic polyurethane (TPU), polyvinylidene fluoride-hexafluoropropylene (PVDF-HFP), and silicone. Successful experimental demonstration of a stretchable electronic circuit in the form of a light-emitting diode (LED) array illustrates the practical suitability of the patterned liquid metal conductors. The developments pave the way for the efficient and economic processing of liquid metal towards scalable manufacturing of stretchable electronic devices and circuits.

\section{RESULTS AND DISCUSSIONS}

\section{Liquid metal patterning in solution-based processes}

Fig. 1a schematically illustrates the key fabrication steps to create liquid metal patterns in a fully solution-based process. A sacrificial shadow mask is a screen printed on a SEBS substrate by using polymeric ink. A thin layer of PDA is subsequently deposited over the entire substrate in an alkaline dopamine solution ${ }^{57}$, followed by dissolving the shadow mask in ethyl alcohol to form the patterned feature. In silver nitrate solution, silver ions are reduced by the catechol groups of PDA to form silver nanoparticles $(\mathrm{NPs})^{57,58}$, which serve as the catalytic nucleation sites to enable the subsequent electroless deposition of $\mathrm{Cu}$ films ${ }^{59,60}$. The liquid metal pattern is obtained by the selective coating of Galinstan over $\mathrm{Cu}$ traces in the presence of dilute hydrochloric $(\mathrm{HCl})$ acid. The $\mathrm{Cu}$ film is gradually dissolved in the liquid metal via alloying reactions, consequently forming a liquid-state conductive feature $\mathrm{e}^{37,61}$. In Fig. 1b, the corresponding optical microscopy images reveal the clear changes in the visual appearance at the key steps in the process, which allows facile identification of the successful deposition of each functional layer. As shown in Fig. 1c, representative liquid metal patterns in the shape of the Sierpinski triangle and sky wheel are created over SEBS substrate, which demonstrates the capability to generate arbitrary and complex features by using this process. In Fig. 1d, the optical microscopy images reveal as-prepared liquid metal lines with various widths from 100 to $300 \mu \mathrm{m}$. The patterning resolution is primarily limited by the zigzag edges commonly encountered in screen-printed patterns, which are formed by squeezing the ink through the nylon meshes ${ }^{62,63}$. Accordingly, the feature resolution and printing quality can be potentially improved by using screens with high 

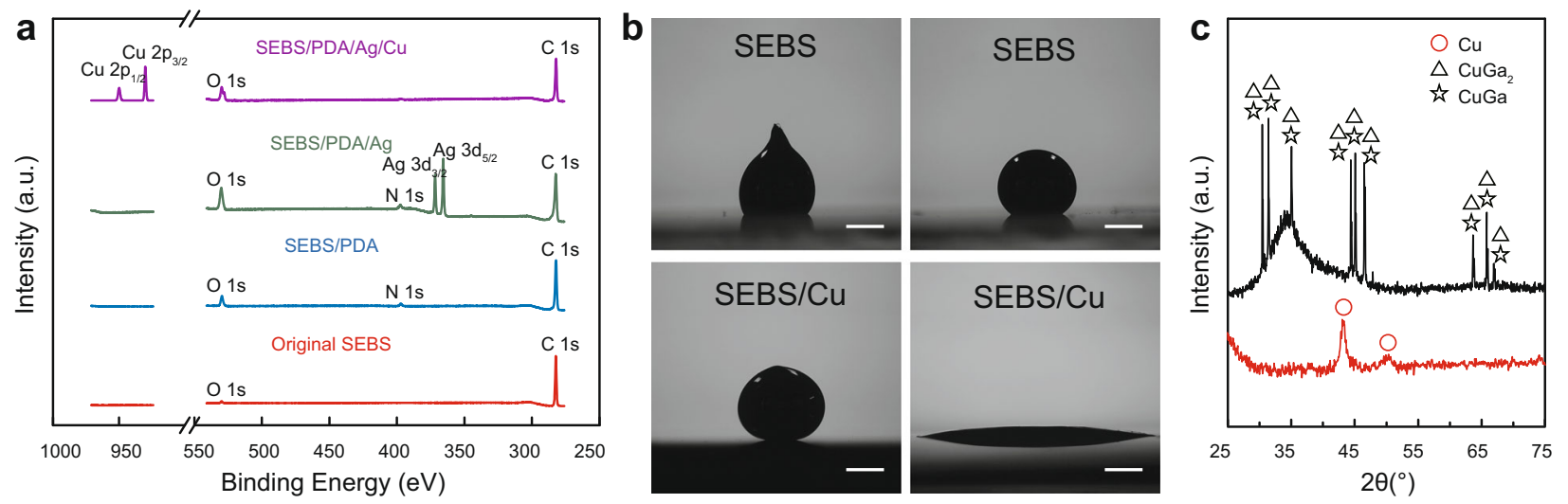

Fig. 2 Characterizations to reveal the patterning mechanism. a XPS spectra acquired from each critical step to achieve electroless deposition of Cu film on SEBS substrate. $\mathbf{b}$ Contact angle images of liquid metal droplets on pristine and Cu-covered SEBS substrates before (left) and after (right) treatment in dilute $\mathrm{HCl}$. Scale bar: $1 \mathrm{~mm}$. c XRD spectra acquired from electroless plated Cu film (red) and liquid metal pattern (black).

mesh counts. In addition, an alternative modified approach by combining the solution-based processes with photolithography allows substantial enhancement in the patterning resolution up to $15 \mu \mathrm{m}$, as demonstrated in Supplementary Fig. 1.

\section{Patterning mechanism}

Additional compositional and morphological characterizations are acquired to decipher the underlying mechanism for the solutionprocessed liquid metal patterning approach. In Fig. 2a, X-ray photoelectron spectroscopy (XPS) analysis reveals the emergence of $\mathrm{N} 1 \mathrm{~s}$ peak at $399.9 \mathrm{eV}$ as a result of simple immersion of SEBS substrate in alkaline dopamine solution ${ }^{57,64}$. After treatment in silver nitrate solution, the formation of Ag seed NPs is verified by the observation of $\mathrm{Ag} \mathrm{3} \mathrm{d}_{3 / 2}(374.3 \mathrm{eV})$ and $\mathrm{Ag} \mathrm{3d_{5/2 }}(368.3 \mathrm{eV}$ ) peaks in the XPS spectrum (green trace) ${ }^{65,66}$. These NPs are sparsely distributed over the PDA film with the dimensions in tens of nanometers, as revealed by the SEM image in Supplementary Fig. 2. These Ag NP seeds further catalyze the subsequent electroless deposition of $\mathrm{Cu}$ film, as confirmed by the $\mathrm{Cu} 2 \mathrm{p}_{1 / 2}$ peak at $952.6 \mathrm{eV}$ and $\mathrm{Cu} 2 \mathrm{p}_{3 / 2}$ peak at $932.8 \mathrm{eV}$ in the spectrum (purple trace) ${ }^{67,68}$. The mussel-inspired PDA coating employed here represents a convenient and versatile platform to activate the SEBS substrate for electroless metallization ${ }^{57}$. In addition, contact angles of Galinstan on different surfaces are further acquired to unravel the mechanism for selective deposition of liquid metal over electroless plated Cu features, as shown in Fig. 2b. Galinstan droplet is passivated by native oxides exhibiting an obvious nonwetting behavior on SEBS substrate with a large contact angle of $123.7^{\circ}$. The contact angle further increases to $135.4^{\circ}$ after removing the surface oxides in dilute $\mathrm{HCl}$ solution. In addition, Galinstan droplets exhibit pronounced nonwetting characteristics with an extremely large contact angle of $160^{\circ}$ on electroless deposited $\mathrm{Cu}$ film under the ambient condition due to nanoscale surface roughness for effective trapping of air pockets (see Supplementary Fig. 3) ${ }^{69,70}$. The contact angle drops to $10.7^{\circ}$ after a short treatment in dilute $\mathrm{HCl}$ solution. The large wettability contrast between the elastomer and $\mathrm{Cu}$ film is the enabler for the selective deposition of non-oxidized liquid metal. In Fig. 2c, the X-ray diffraction (XRD) spectrum of electroless deposited $\mathrm{Cu}$ features reveals two broad peaks at $43.3^{\circ}$ and $50.5^{\circ}$ corresponding to $\mathrm{Cu}$ with fine grains $\mathrm{s}^{71,72}$. After the deposition of liquid metal, a series of crystalline peaks emerge in XRD spectrum as a result of the formation of intermetallic compounds of CuGa and $\mathrm{CuGa}_{2}{ }^{37,61}$. The alloying reaction at the interface is essentially the driving force for the notably improved wettability with non-oxidized liquid metal ${ }^{73}$. The electroless deposited $\mathrm{Cu}$ film, therefore, serves as an interfacial layer to promote the adhesion and the spread of liquid metal. In addition, the alloying process also accounts for the gradual dissolution of $\mathrm{Cu}$ film underneath the liquid metal feature (see Supplementary Fig. 4), consequently producing a liquid-state suspension containing precipitated crystals of intermetallic compounds $s^{37,61}$. The average dimension of the fine precipitates is determined as $\sim 50 \mathrm{~nm}$ according to the Scherrer equation, which exhibits limited influences on the deformability of the liquid metal features. After the patterning process, the spontaneously formed native oxides on liquid metal features effectively function as solid shells to retain structural stability.

\section{Physical properties of liquid metal features}

The as-prepared liquid metal patterns exhibit a high electrical conductivity of $4.15 \times 10^{4} \mathrm{~S} \mathrm{~cm}^{-1}$, The value is in fact slightly higher than that of bulk Galinstan $\left(3.46 \times 10^{4} \mathrm{~S} \mathrm{~cm}^{-1}\right)^{29,30}$, as a result of the formation of highly conductive intermetallic compounds of $\mathrm{CuGa}$ and $\mathrm{CuGa}_{2}{ }^{61}$. The liquid metal features exhibit excellent environmental stability to retain the high conductivity over long-term storage under ambient conditions, as shown in Supplementary Fig. 5. As regards mechanical deformability, Fig. 3a presents the optical images of arrays of liquid metal lines on SEBS substrate under uniaxial stretching with different tensile strains. The liquid metal feature is highly stretchable to retain the intact morphology at a giant strain of $1000 \%$. The normalized resistance as a function of the uniaxial tensile strain is shown in Fig. 3b. The liquid metal conductor exhibits increased resistance by 1.5 times at $100 \%$ strain, 15 times at $500 \%$ strain, and 48 times at $1000 \%$ strain, respectively. The exceptional stretchability is a unique feature of liquid-state conductors capable of accommodating enormous tensile strains. A summary of the key characteristics of various stretchable conductors is provided in Supplementary Table 1 to highlight the attractive properties of the solution-processed liquid metal conductor. In addition, the liquid metal conductor is accessed by strain-controlled fatigue tests, as illustrated in Fig. 3c. The resistance shows negligible change over 1000 stretch-relaxation cycles to $300 \%$ strain, which demonstrates excellent durability as an attractive attribute for the long-term implementations under practical settings.

\section{Fabrication of liquid metal features on various stretchable substrates}

Liquid metal is naturally oxidized under ambient conditions and shows nonwetting characteristics on a variety of stretchable substrates (see Supplementary Fig. 6). The passivating surface oxides are conveniently removed by treatment in dilute $\mathrm{HCl}$ 
a
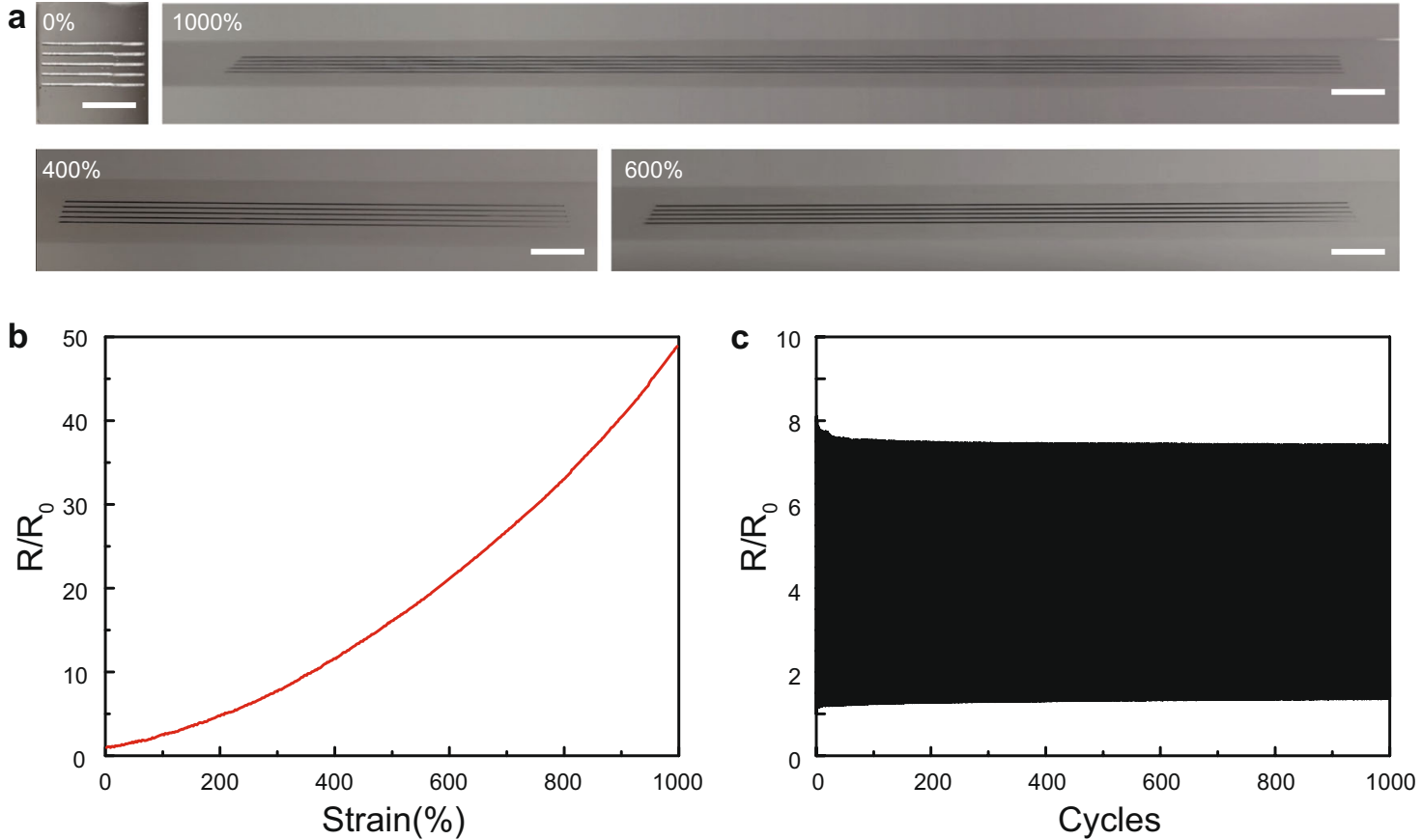

Fig. 3 Electromechanical properties of liquid metal features. a Optical images of a patterned liquid metal feature at pristine and uniaxially stretched states up to $1000 \%$ strain. Scale bar: $1 \mathrm{~cm}$. b Normalized resistance as a function of tensile strain. c Evolution of normalized resistance during 1000 stretch-relaxation cycles to $300 \%$ strain.

a
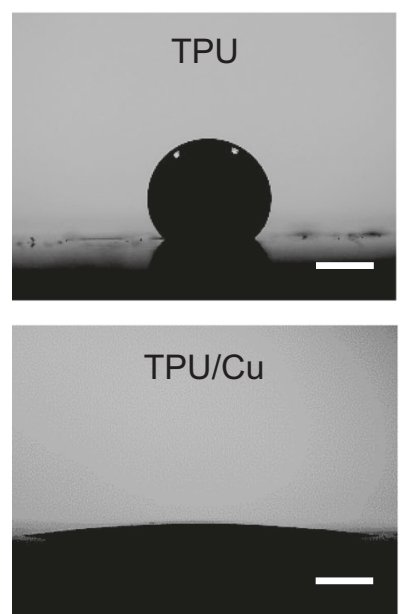

b

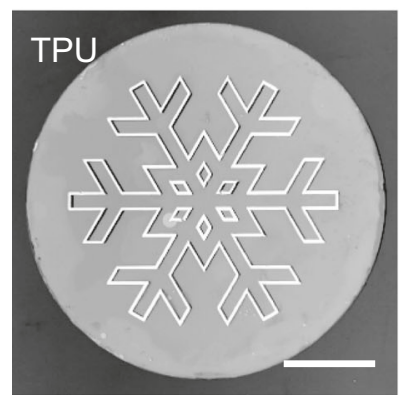

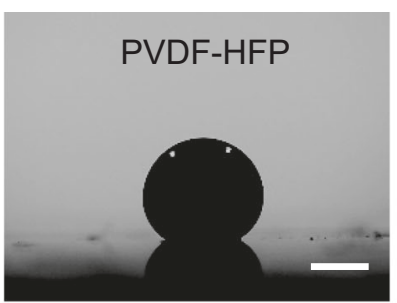
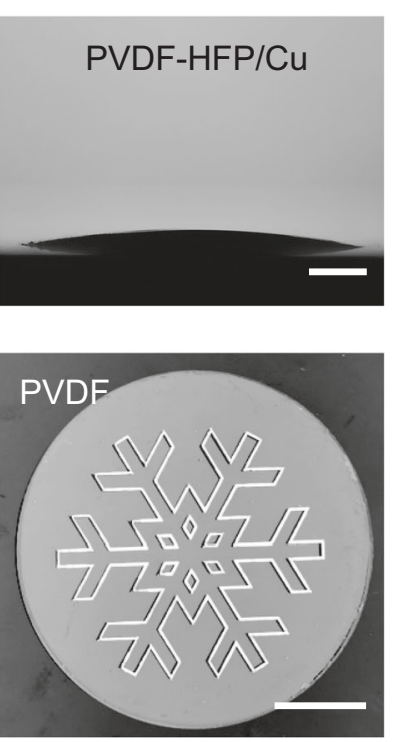
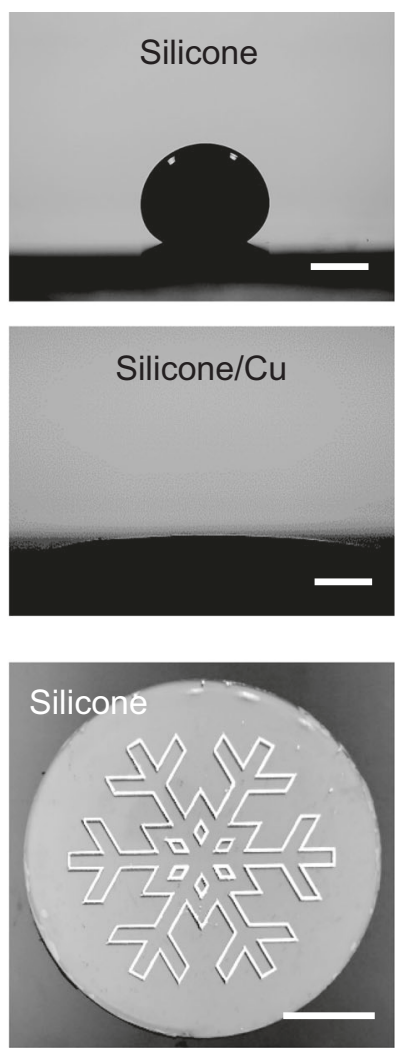

Fig. 4 Liquid metal patterning on various stretchable substrates. a Contact angle images of non-oxidized liquid metal droplets on different substrates at the pristine state (top) and after electroless deposition of Cu films (bottom). Scale bar: $1 \mathrm{~mm}$. b Optical images of snowflakeshaped liquid metal patterns prepared on different substrates. Scale bar: $2 \mathrm{~cm}$. 

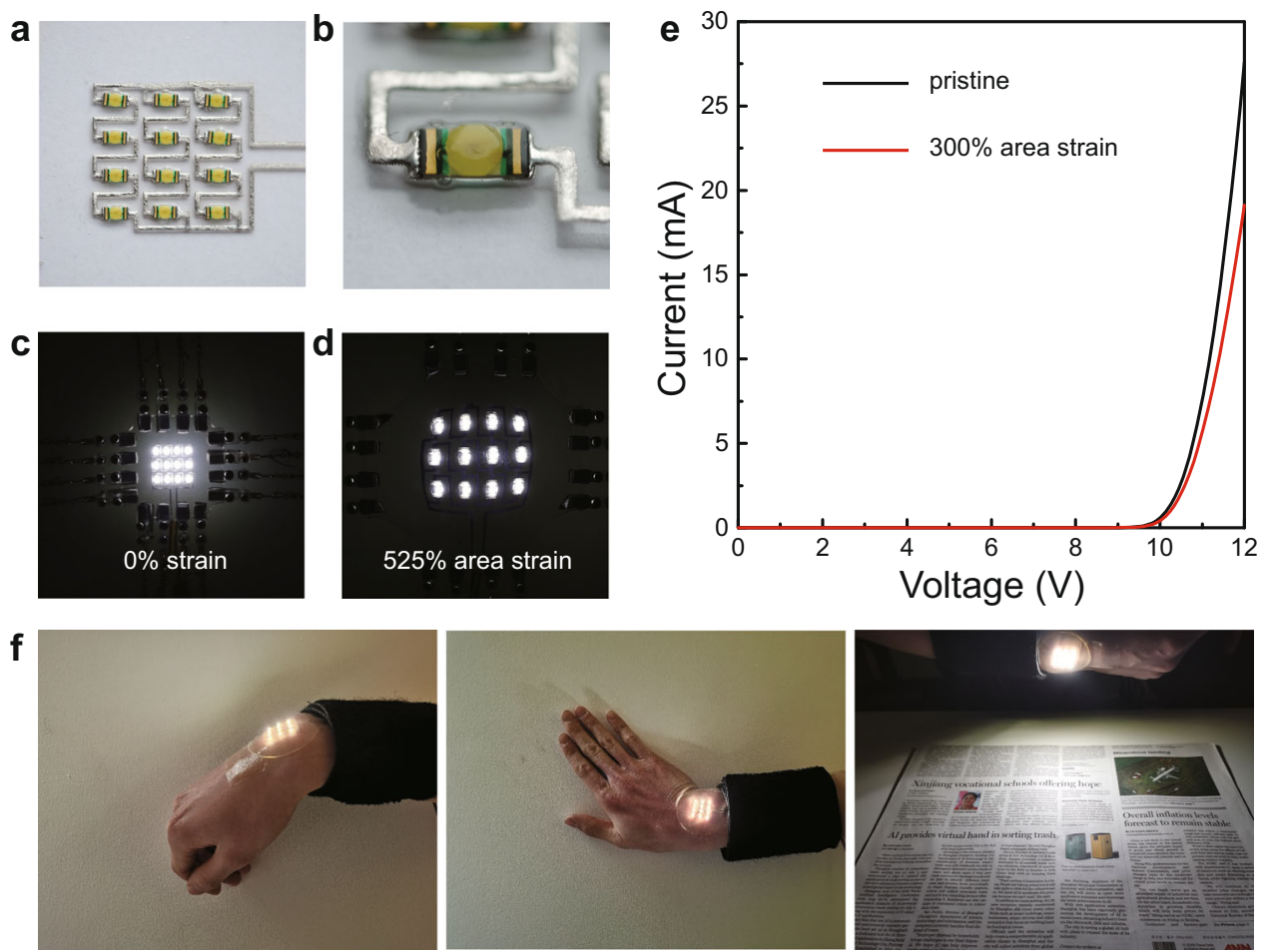

Fig. 5 Liquid metal-based stretchable integrated circuit systems. a Optical images of a stretchable integrated circuit in the form of an LED matrix. b Optical microscopy image revealing a representative LED chip mounted onto liquid metal interconnects. c, $\mathbf{d}$ Optical images of a luminous LED matrix at the relaxed state (c) and biaxially stretched state with $525 \%$ area strain (d). e Current-voltage curves of the LED matrix at 0 and $300 \%$ area strains. f Optical images of the LED matrix attached to the wrist as a wearable light source under wrist flexion (left), and ulnar deviation (middle) gestures, which effectively lights up newspapers under dim lighting conditions (right).

solution. A key enabler for the solution-processed liquid metal pattern is the large wettability contrast between copper and elastomer surfaces. In addition to SEBS, non-oxidized Galinstan droplets lack the wettability with additional elastomer substrates, manifested as large contact angles of $129.7^{\circ}$ on TPU, $136.1^{\circ}$ on PVDF-HFP, and $134.9^{\circ}$ on silicone (see Fig. $4 a$ and Supplementary Table 2). The slightly different contact angles are possibly associated with variations in surface functional groups and interfacial energies ${ }^{40,74}$. Subsequently, the self-polymerization of dopamine is a generic process to modify these elastomeric substrates with surface adherent PDA films. In Supplementary Fig. 7, XPS spectra reveal $N$ 1s peak at the binding energy of $399.9 \mathrm{eV}$ as the evidence for successful deposition of PDA films, which promotes the electroless deposition of copper films on these elastomers. The Cu films show a strong affinity with nonoxidized gallium irrespectively of the underlying substrates (see Fig. 4a and Supplementary Table 3). The sharp contrasts in wettability allow selective depositions of liquid metal patterns by using $\mathrm{Cu}$ templates on the elastomer substrates. In Fig. 4b, snowflake-shaped liquid metal features are successfully created by the solution process over TPU, PVDF-HFP, and silicone substrates, which demonstrates the technique as a generic approach for scalable fabrication of high-fidelity patterns. The as-prepared liquid metal features show ultrahigh stretchability on all these substrates (see Supplementary Fig. 8). The elastomer choice shows pronounced influences on the electromechanical properties because of the different responses to tensile deformations (see Supplementary Fig. 9). Although liquid metal patterning by selective wetting has been demonstrated previously ${ }^{15,32,53}$, the physical vapor deposition of solid $\mathrm{Au}$ and $\mathrm{Cu}$ films over elastomeric substrates requires tailored recipes to prevent crack formations due to the compressive stress developed during the deposition process involving elevated temperatures $32,55,56$. In contrast, the fully solution-based process demonstrated here is carried out under ambient conditions and compatible with various substrates, which opens up an attractive avenue for the facile deployment of liquid metal conductors for stretchable and wearable electronics.

\section{Implementations in stretchable integrated circuit systems}

As regards the practical implementations, the exceptional deformability of liquid metal conductor allows heterogeneous integration of off-the-shelf chips on elastomer substrates as stretchable integrated circuit systems. A representative system in the form of a LED matrix is shown in Fig. 5a. Briefly, the liquid metal circuit pattern containing interconnects and contact pads is created on SEBS substrate by the solution process. LED chips are mechanically soldered to the contact pads by gentle pressing with a tweezer ${ }^{47}$. The fabrication is completed by ultrasonic spray deposition of a layer of SEBS for encapsulation. The as-prepared circuit system is fully functional to provide bright emissions at the relaxed state (see Fig. $5 \mathrm{c}$ ) and highly stretchable to retain a uniform luminous pattern at a large area strain of 525\% (see Fig. $5 \mathrm{~d}$ ). Figure 5 e shows the current-voltage curves at the relaxed and stretched $(300 \%$ area strain) states, respectively. The minor decrease in the current at the stretched state is associated with the increased resistance of liquid metal interconnects, which suggests the high conductivity of the liquid metal circuit is sufficient to retain the fairly stable performance of the system under highly deformed conditions. The dynamic process to repetitively deform the LED array to an area strain of $300 \%$ is shown in Supplementary Movie S1, which effectively illustrates the durability of the system. The compliant mechanical properties allow the soft LED array to be directly mounted on the wrist by using silicone-based skin adhesive. The circuit system functions as a wearable lighting device while the user makes different hand gestures, which provides sufficient emissions to light up the 
newspapers under a dim lighting environment (see Fig. 5f). The skin-like circuit system exhibits excellent deformability and resilience for the flexible delivery of various electronic functionalities to the human body.

In summary, we have established a solution processing approach to generate delicate patterns of liquid metal over elastomer substrates. As deposited liquid metal features exhibit bulk-level conductivity, ultrahigh deformability, and repetitive stretchability. The scalable and economic process is carried out under ambient conditions and is compatible with various elastomeric substrates. The fabrication of a stretchable circuit system of an LED array illustrates the practical implementation of liquid metal conductors for the heterogeneous integration of chipscale components over elastomeric substrates. The facile creation of highly conductive and deformable features demonstrated in this study may stimulate the development of a broad range of stretchable electronic devices and systems.

\section{METHODS}

\section{Materials and preparations}

All elastomers used in this study are commercially available including hydrogenated styrene-ethylene-butylene-styrene (SEBS, Tuftec H1221) from Asahi Kasei Corporation, TPU (Tecothane AR-62A) from Lubrizol Inc., PVDF-HFP (DYNEON FC2176) from 3M Co. of the United States, silicone (Dragon Skin 20) from Smooth-On, Inc. Fumed silica (AEROSIL R974) was acquired from Evonik Degussa GmbH (Shanghai, China). Other chemical reagents were purchased from Shanghai Macklin Biochemical Co., Ltd. without further purification. Galinstan was prepared by melting a mixture of metal pieces of $\mathrm{Ga}$, In, and $\mathrm{Sn}$ in a weight ratio of $68.5: 21.5: 10$ at $80^{\circ} \mathrm{C}$ for $2 \mathrm{~h}$ in a glovebox. As regards the preparation of stretchable substrates, all thermoplastic elastomers in the form of rubbery solids were dissolved in selected solvents and then drop cast onto nonsticky glass wafers functionalized with OTS, followed by natural evaporation to thoroughly remove the solvents. The solvent choices and concentrations were tailored for optimized quality of the resulting substrates, including toluene for SEBS $(20 \mathrm{w} / \mathrm{v} \%)$, tetrahydrofuran for TPU (20 w/v \%), and 4-methyl-2-pentanone for PVDF-HFP ( $25 \mathrm{w} / \mathrm{v} \%)$. The liquid precursors of Dragon Skin 20 were thoroughly mixed and then drop cast onto nonsticky glass wafers. Silicone substrates were obtained after thermal curing at $120^{\circ} \mathrm{C}$ in an oven for $2 \mathrm{~h}$.

\section{Fabrication of patterned liquid metal features}

The printable ink for the sacrificial mask was formulated by dissolving PVB $(10 \mathrm{w} / \mathrm{v} \%)$ and fumed silica $(4 \mathrm{w} / \mathrm{v} \%)$ in ethyl alcohol. Sacrificial masks were generated on elastomeric substrates by using a manual screen printer with a 420-mesh nylon screen. The substrates were submerged in a solution of dopamine hydrochloride $(2 \mathrm{mg} / \mathrm{mL})$ in $50 \mathrm{mM}$ Tris buffer $(\mathrm{pH}$ 8.5) for deposition of PDA, which was proceeded over $14 \mathrm{~h}$ under constant shaking. Subsequently, the substrates were washed with deionized water and then rinsed in ethyl alcohol to remove the sacrificial mask. The substrates were submerged into silver nitrate aqueous solution $(0.2 \mathrm{M})$ for $3 \mathrm{~h}$, which promotes the growth of Ag NPs as the seed layer. The substrates were then submerged into the chemical bath for $\mathrm{Cu}$ electroless deposition, which consist of $0.0258 \mathrm{M}$ copper sulfate pentahydrate, $0.0258 \mathrm{M}$ edetate sodium, $0.268 \mathrm{M}$ triethanolamine, $0.09 \mathrm{M}$ sulfuric acid, $0.1 \mathrm{w} / \mathrm{w} \%$ Triton $\mathrm{X}-$ 100 , and $0.074 \mathrm{M}$ dimethylamine borane. The regions containing silver NP seeds were deposited with a layer of $\sim 1-\mu \mathrm{m}$-thick copper film after a typical reaction for $30 \mathrm{~min}$. A layer of liquid metal was selectively deposited onto the patterned $\mathrm{Cu}$ film by rolling bulk Galinstan over the entire substrate submerged in dilute hydrogen chloride solution ( $4 \mathrm{w} / \mathrm{v} \%)$. After the gradual dissolution of the $\mathrm{Cu}$ film in liquid metal through alloying reactions, compliant conductive features in the liquid state were formed on the elastomeric substrate. The thickness of the $\mathrm{Cu}$ film was fairly thin to ensure the excellent deformability of the liquid metal with a small percentage of incorporated alloys ${ }^{61}$. As regards the photolithographybased pattering process, the scarificial mask was replaced with photoresist film (AZ P4620) patterned by using a lithography machine (SUSS MA6). The developer was obtained by mixing Developer (AZ $400 \mathrm{~K}$ ) in deionized water at a volume ratio of 1:3. The patterned features and detailed procedures were shown in Supplementary Fig. 1.

\section{Material characterizations}

Structural characterization and compositional analysis were carried out by optical microscopy (Keyence VHX-6000 digital microscope), confocal laser scanning microscopy (a Keyence VK-X1000 microscope), SEM (Zeiss GeminiSEM 500 field emission scanning electron microscope), XRD (Rigaku Ultima III X-ray diffractometer), and XPS (PHI Quantera II scanning XPS microprobe). Optical images and movies were acquired using a Fujifilm $\mathrm{X}$-T10 camera. The wettability of Galinstan droplets on elastomers and chemical deposited $\mathrm{Cu}$ films was analyzed by a contact angle goniometer (SDC-200, Dongguan Sindin Precision Instrument Co., Ltd.). The mechanical properties were analyzed using a universal testing machine (Shimadzu AGS-X) equipped with a $50 \mathrm{~N}$ load cell. The sheet resistance was measured with a four-point configuration by using a Keithley 2110 digital multimeter and a GWINSTEK GOM-805 milliohm meter. The change in the resistance in response to the tensile strain was evaluated on a homemade motorized linear stage.

\section{Fabrication and evaluation for stretchable LED arrays}

A liquid metal circuit pattern was created on SEBS substrate based on the solution process. LED chips (LTW-216TS5, 1206, Lite-On Inc.) were positioned on the contact pads and gently pressed with a tweezer for reliable electrical connections ${ }^{47}$. A layer of SEBS was deposited over the sample from its solution in toluene $(4 \mathrm{w} / \mathrm{v} \%)$ for encapsulation, which was carried out in an ultrasonic spray coating machine operated at $40 \mathrm{kHz}$ (DWF40-60, Hangzhou Dowell Ultrasonic Technology, Co., Ltd). The as-prepared stretchable LED array was powered by an HYELEC DC power supply HY3005ET. The electrical characteristics were measured by a Keithley $2634 \mathrm{~B}$ sourcemeter. The stretchability of the LED array was evaluated on a homemade biaxial linear stage.

\section{DATA AVAILABILITY}

All data needed to evaluate the conclusions in the paper are present in the paper and/or the Supplementary Materials. Additional data related to this paper may be requested from the authors.

Received: 3 June 2021; Accepted: 1 September 2021; Published online: 23 September 2021

\section{REFERENCES}

1. Rogers, J. A., Someya, T. \& Huang, Y. Materials and mechanics for stretchable electronics. Science 327, 1603-1607 (2010).

2. Choi, S., Lee, H., Ghaffari, R., Hyeon, T. \& Kim, D.-H. Recent advances in flexible and stretchable bio-electronic devices integrated with nanomaterials. Adv. Mater. 28, 4203-4218 (2016).

3. Suo, Z. Mechanics of stretchable electronics and soft machines. MRS Bull. 37, 218-225 (2012).

4. Wang, S., Oh, J. Y., Xu, J., Tran, H. \& Bao, Z. Skin-inspired electronics: an emerging paradigm. Acc. Chem. Res. 51, 1033-1045 (2018).

5. Chung, H. U. et al. Binodal, wireless epidermal electronic systems with in-sensor analytics for neonatal intensive care. Science 363, eaau0780 (2019).

6. Chen, Y. et al. Skin-like biosensor system via electrochemical channels for noninvasive blood glucose monitoring. Sci. Adv. 3, e1701629 (2017).

7. Son, D. et al. Multifunctional wearable devices for diagnosis and therapy of movement disorders. Nat. Nanotechnol. 9, 397-404 (2014).

8. Wang, X., Gu, Y., Xiong, Z., Cui, Z. \& Zhang, T. Silk-molded flexible, ultrasensitive, and highly stable electronic skin for monitoring human physiological signals. Adv. Mater. 26, 1336-1342 (2014).

9. Kim, J. et al. Stretchable silicon nanoribbon electronics for skin prosthesis. Nat. Commun. 5, 5747 (2014).

10. Chortos, A., Liu, J. \& Bao, Z. Pursuing prosthetic electronic skin. Nat. Mater. 15 , 937-950 (2016).

11. Jeong, J.-W. et al. Materials and optimized designs for human-machine interfaces via epidermal electronics. Adv. Mater. 25, 6839-6846 (2013).

12. Jin, $\mathrm{H}$. et al. Enhancing the performance of stretchable conductors for E-textiles by controlled Ink permeation. Adv. Mater. 29, 1605848 (2017).

13. Wang, M. et al. Gesture recognition using a bioinspired learning architecture that integrates visual data with somatosensory data from stretchable sensors. Nat. Electron. 3, 563-570 (2020).

14. Matsuhisa, N., Chen, X., Bao, Z. \& Someya, T. Materials and structural designs of stretchable conductors. Chem. Soc. Rev. 48, 2946-2966 (2019). 
15. Ozutemiz, K. B., Wissman, J., Ozdoganlar, O. B. \& Majidi, C. EGaln-metal interfacing for liquid metal circuitry and microelectronics integration. Adv. Mater. Interfaces 5, 1701596 (2018).

16. Liu, S., Shah, D. S. \& Kramer-Bottiglio, R. Highly stretchable multilayer electronic circuits using biphasic gallium-indium. Nat. Mater. 20, 851-858 (2021).

17. Chun, K.-Y. et al. Highly conductive, printable and stretchable composite films of carbon nanotubes and silver. Nat. Nanotechnol. 5, 853-857 (2010).

18. Xu, F. \& Zhu, Y. Highly conductive and stretchable silver nanowire conductors. Adv. Mater. 24, 5117-5122 (2012).

19. Matsuhisa, N. et al. Printable elastic conductors by in situ formation of silver nanoparticles from silver flakes. Nat. Mater. 16, 834-840 (2017).

20. Oh, J. Y., Kim, S., Baik, H.-K. \& Jeong, U. Conducting polymer dough for deformable electronics. Adv. Mater. 28, 4455-4461 (2016).

21. Wang, Y. et al. A highly stretchable, transparent, and conductive polymer. Sci. Adv. 3, e1602076 (2017).

22. Savagatrup, S. et al. Plasticization of PEDOT:PSS by common additives for mechanically robust organic solar cells and wearable sensors. Adv. Funct. Mater. 25, 427-436 (2015).

23. Keplinger, C. et al. Stretchable, transparent, ionic conductors. Science $\mathbf{3 4 1}$ 984-987 (2013)

24. Larson, C. et al. Highly stretchable electroluminescent skin for optical signaling and tactile sensing. Science 351, 1071-1074 (2016).

25. Chossat, J., Park, Y., Wood, R. J. \& Duchaine, V. A Soft Strain Sensor Based on lonic and Metal Liquids. IEEE Sens. J. 13, 3405-3414 (2013).

26. Ota, H. et al. Highly deformable liquid-state heterojunction sensors. Nat. Commun. 5, 5032 (2014).

27. Jia, H., He, Y., Zhang, X., Du, W. \& Wang, Y. Integrating ultra-thermal-sensitive fluids into elastomers for multifunctional flexible sensors. Adv. Electron. Mater. 1, 1500029 (2015)

28. Zhu, S. et al. Ultrastretchable fibers with metallic conductivity using a liquid metal alloy core. Adv. Funct. Mater. 23, 2308-2314 (2013).

29. Joshipura, I. D., Ayers, H. R., Majidi, C. \& Dickey, M. D. Methods to pattern liquid metals. J. Mater. Chem. C. 3, 3834-3841 (2015)

30. Dickey, M. D. Stretchable and soft electronics using liquid metals. Adv. Mater. 29, 1606425 (2017).

31. Kubo, M. et al. Stretchable microfluidic radiofrequency antennas. Adv. Mater. 22, 2749-2752 (2010).

32. Jeong, Y. R. et al. A skin-attachable, stretchable integrated system based on liquid GalnSn for wireless human motion monitoring with multi-site sensing capabilities. NPG Asia Mater. 9, e443-e443 (2017).

33. Wang, Y. et al. Printable liquid-metal@PDMS stretchable heater with high stretchability and dynamic stability for wearable thermotherapy. Adv. Mater. Technol. 4, 1800435 (2019).

34. Guan, L. et al. Stretchable-fiber-confined wetting conductive liquids as wearable human health monitors. Adv. Funct. Mater. 26, 4511-4517 (2016).

35. Park, Y., Chen, B. \& Wood, R. J. Design and fabrication of soft artificial skin using embedded microchannels and liquid conductors. IEEE Sens. J. 12, 2711-2718 (2012).

36. Gao, Y. et al. Wearable microfluidic diaphragm pressure sensor for health and tactile touch monitoring. Adv. Mater. 29, 1701985 (2017).

37. Li, Y., Feng, S., Cao, S., Zhang, J. \& Kong, D. Printable liquid metal microparticle ink for ultrastretchable electronics. ACS Appl. Mater. Interfaces 12, 50852-50859 (2020).

38. Dickey, M. D. et al. Eutectic gallium-indium (EGaln): a liquid metal alloy for the formation of stable structures in microchannels at room temperature. Adv. Funct. Mater. 18, 1097-1104 (2008).

39. Lin, Y. et al. Vacuum filling of complex microchannels with liquid metal. Lab Chip 17, 3043-3050 (2017)

40. Li, G., Parmar, M. \& Lee, D.-W. An oxidized liquid metal-based microfluidic platform for tunable electronic device applications. Lab Chip 15, 766-775 (2015).

41. Wu, S.-Y., Yang, C., Hsu, W. \& Lin, L. 3D-printed microelectronics for integrated circuitry and passive wireless sensors. Microsyst. Nanoeng. 1, 15013 (2015).

42. Andrews, J. B. et al. Patterned liquid metal contacts for printed carbon nanotube transistors. ACS Nano 12, 5482-5488 (2018).

43. Wang, J. et al. Omnidirectional printing of soft elastomer for liquid-state stretchable electronics. ACS Appl. Mater. Interfaces 11, 18590-18598 (2019).

44. Boley, J. W., White, E. L., Chiu, G. T. C. \& Kramer, R. K. Direct writing of galliumindium alloy for stretchable electronics. Adv. Funct. Mater. 24, 3501-3507 (2014).

45. Park, Y.-G., An, H. S., Kim, J.-Y. \& Park, J.-U. High-resolution, reconfigurable printing of liquid metals with three-dimensional structures. Sci. Adv. 5, eaaw2844 (2019).

46. Chen, S., Wang, H., Zhao, R., Rao, W. \& Liu, J. Liquid metal composites. Matter 2 1446-1480 (2020)

47. Zhang, K., Kong, S., Li, Y., Lu, M. \& Kong, D. Soft elastomeric composite materials with skin-inspired mechanical properties for stretchable electronic circuits. Lab Chip 19, 2709-2717 (2019).
48. Yan, J. et al. Solution processable liquid metal nanodroplets by surface-initiated atom transfer radical polymerization. Nat. Nanotechnol. 14, 684-690 (2019).

49. Hohman, J. et al. Directing substrate morphology via self-assembly: ligandmediated scission of gallium-indium microspheres to the nanoscale. Nano Lett. 11, 5104-5110 (2011)

50. Liu, S. et al. Laser sintering of liquid metal nanoparticles for scalable manufacturing of soft and flexible electronics. ACS Appl. Mater. Interfaces 10, 28232-28241 (2018)

51. Tang, L., Mou, L., Zhang, W. \& Jiang, X. Large-scale fabrication of highly elastic conductors on a broad range of surfaces. ACS Appl. Mater. Interfaces 11, 7138-7147 (2019).

52. Boley, J. W., White, E. L. \& Kramer, R. K. Mechanically sintered gallium-indium nanoparticles. Adv. Mater. 27, 2355-2360 (2015).

53. Li, G., Wu, X. \& Lee, D.-W. Selectively plated stretchable liquid metal wires for transparent electronics. Sens. Actuators B Chem. 221, 1114-1119 (2015).

54. Tang, J., Zhao, X., Li, J., Zhou, Y. \& Liu, J. Liquid metal phagocytosis: intermetallic wetting induced particle internalization. Adv. Sci. 4, 1700024 (2017).

55. Bowden, N., Brittain, S., Evans, A. G., Hutchinson, J. W. \& Whitesides, G. M. Spontaneous formation of ordered structures in thin films of metals supported on an elastomeric polymer. Nature 393, 146-149 (1998).

56. Yu, S., Sun, Y., Ni, Y., Zhang, X. \& Zhou, H. Controlled formation of surface patterns in metal films deposited on elasticity-gradient PDMS substrates. ACS Appl. Mater. Interfaces 8, 5706-5714 (2016).

57. Lee, H., Dellatore, S. M., Miller, W. M. \& Messersmith, P. B. Mussel-inspired surface chemistry for multifunctional coatings. Science 318, 426 (2007).

58. Zhang, L. et al. Combination of bioinspiration: a general route to superhydrophobic particles. J. Am. Chem. Soc. 134, 9879-9881 (2012).

59. Lee, C.-L. \& Syu, C.-C. Ag nanoparticle as a new activator for catalyzing electroless copper bath with 2,2'-bipyridyl. Electrochim. Acta 56, 8880-8883 (2011).

60. Hsu, P.-C. et al. Electrolessly deposited electrospun metal nanowire transparent electrodes. J. Am. Chem. Soc. 136, 10593-10596 (2014).

61. Tang, J. et al. Gallium-based liquid metal amalgams: transitional-state metallic mixtures (TransM2ixes) with enhanced and tunable electrical, thermal, and mechanical properties. ACS Appl. Mater. Interfaces 9, 35977-35987 (2017).

62. Zhou, Y. et al. Stretchable high-permittivity nanocomposites for epidermal alternating-current electroluminescent displays. ACS Mater. Lett. 1, 511-518 (2019).

63. Zhao, C. et al. Fully screen-printed, multicolor, and stretchable electroluminescent displays for epidermal electronics. ACS Appl. Mater. Interfaces 12, 47902-47910 (2020).

64. Zhao, L., Chen, D. \& Hu, W. Patterning of metal films on arbitrary substrates by using polydopamine as a UV-sensitive catalytic layer for electroless deposition. Langmuir 32, 5285-5290 (2016).

65. Han, S. W., Kim, Y. \& Kim, K. Dodecanethiol-derivatized Au/Ag bimetallic nanoparticles: TEM, UV/VIS, XPS, and FTIR analysis. J. Colloid Interface Sci. 208, 272-278 (1998).

66. Kato, Y. et al. Silver iodide formation in methyl ammonium lead iodide perovskite solar cells with silver top electrodes. Adv. Mater. Interfaces 2, 1500195 (2015).

67. Yu, J., Zhang, J. \& Liu, S. lon-exchange synthesis and enhanced visible-light photoactivity of CuS/ZnS nanocomposite hollow spheres. J. Phys. Chem. C. 114 13642-13649 (2010).

68. Liu, S. et al. High-performance flexible quasi-solid-state supercapacitors realized by molybdenum dioxide@nitrogen-doped carbon and copper cobalt sulfide tubular nanostructures. Adv. Sci. 5, 1800733 (2018)

69. Yang, C., Tartaglino, U. \& Persson, B. N. J. Influence of surface roughness on superhydrophobicity. Phys. Rev. Lett. 97, 116103 (2006).

70. Nosonovsky, M. \& Bhushan, B. Roughness-induced superhydrophobicity: a way to design non-adhesive surfaces. J. Phys.: Condens. Matter 20, 225009 (2008).

71. Wu, S.-H. \& Chen, D.-H. Synthesis of high-concentration $\mathrm{Cu}$ nanoparticles in aqueous CTAB solutions. J. Colloid Interface Sci. 273, 165-169 (2004).

72. Lv, W. et al. Electrodeposition of nano-sized bismuth on copper foil as electrocatalyst for reduction of CO2 to formate. Appl. Surf. Sci. 393, 191-196 (2017).

73. Cui, Y. et al. Metallic bond-enabled wetting behavior at the liquid $\mathrm{Ga} / \mathrm{CuGa} 2$ interfaces. ACS Appl. Mater. Interfaces 10, 9203-9210 (2018).

74. Decker, E. L., Frank, B., Suo, Y. \& Garoff, S. Physics of contact angle measurement. Colloid Surf. A 156, 177-189 (1999).

\section{ACKNOWLEDGEMENTS}

This work was supported by the Key Research and Development Program of Jiangsu Provincial Department of Science and Technology of China (Grant No. BE2019002) and the High-Level Entrepreneurial and Innovative Talents Program of Jiangsu Province. 


\section{AUTHOR CONTRIBUTIONS}

D.K. conceived the original idea of the project. H.Z. carried out material preparations, device fabrications, and characterizations. S.W. participated in the device fabrications and electrical measurements. M.Z. optimized the screen-printing parameters. T.L. and G.H. carried out the photolithography. H.Z. and D.K. wrote the original manuscript. All authors contributed to the scientific planning and discussions.

\section{COMPETING INTERESTS}

The authors declare no competing interests.

\section{ADDITIONAL INFORMATION}

Supplementary information The online version contains supplementary material available at https://doi.org/10.1038/s41528-021-00123-x.

Correspondence and requests for materials should be addressed to Desheng Kong.

Reprints and permission information is available at http://www.nature.com/ reprints
Publisher's note Springer Nature remains neutral with regard to jurisdictional claims in published maps and institutional affiliations.

Open Access This article is licensed under a Creative Commons Attribution 4.0 International License, which permits use, sharing, adaptation, distribution and reproduction in any medium or format, as long as you give appropriate credit to the original author(s) and the source, provide a link to the Creative Commons license, and indicate if changes were made. The images or other third party material in this article are included in the article's Creative Commons license, unless indicated otherwise in a credit line to the material. If material is not included in the article's Creative Commons license and your intended use is not permitted by statutory regulation or exceeds the permitted use, you will need to obtain permission directly from the copyright holder. To view a copy of this license, visit http://creativecommons. org/licenses/by/4.0/.

(c) The Author(s) 2021 